\title{
ELLIPTIC PSEUDO DIFFERENTIAL OPERATORS DEGENERATE ON A SYMPLECTIC SUBMANIFOLD
}

\author{
BY BERNARD HELFFER AND LUIGI RODINO \\ Communicated by Robert T. Seeley, April 12, 1976
}

1. Introduction. This note is concerned with the classes of pseudo differential operators $L^{m, M}(\Omega, \Sigma), \Sigma$ symplectic submanifold of codimension 2, in Sjöstrand [4] ; the definitions of $P$ in $L^{m, M}(\Omega, \Sigma)$ and of the associated winding number $N$ are recalled in $\S 2$. In Helffer [2] the study of the hypoellipticity of $P$ is reduced to the analysis of the bounded solutions of an ordinary differential equation. Here we deduce an explicit result for $N=2-M$ : essentially, we can prove that in this case all the bounded solutions are products of an exponential function with polynomials.

2. The classes $L^{m, M}(\Omega, \Sigma)$ and the winding number. Let $\Omega \subset \mathbf{R}^{n}$ be an open set. Let $\Sigma \subset T^{*}(\Omega) \backslash 0$ be a closed conic symplectic submanifold of codimension 2 ( $\Sigma$ symplectic means that the restriction of the symplectic form $\omega=\Sigma d \xi_{s} \wedge d x_{s}$ to $\Sigma$ is nondegenerate $) . L^{m, M}(\Omega, \Sigma)$ is the set of all the pseudo differential operators $P$ which have a symbol of the form

$$
p(x, \xi) \sim \sum_{j=0}^{\infty} p_{m-j / 2}(x, \xi)
$$

where $p_{m-j / 2}$ is positively homogeneous of degree $m-j / 2$ and for every $K \subset \subset$ $\Omega$ there exists a constant $C_{K}$ such that

$$
\left|p_{m-j / 2}(x, \xi)\right| /|\xi|^{m-j / 2} \leqslant C_{K} d_{\Sigma}^{M-j}(x, \xi), \quad 0 \leqslant j \leqslant M
$$

for all $(x, \xi) \in K \times \mathbf{R}^{n},|\xi|>1\left(d_{\Sigma}(x, \xi)\right.$ is the distance from $(x, \xi /|\xi|)$ to $\left.\Sigma\right)$.

Fix $\rho$ in $\Sigma$, denote by $N_{\rho}(\Sigma)$ the orthogonal space of $T_{\rho}(\Sigma)$ with respect to $\omega$ and choose two linear coordinates on $N_{\rho}(\Sigma) u_{1}, u_{2}$ such that $\omega / N_{\rho}(\Sigma)=$ $d u_{2} \wedge d u_{1}$. Take $X=\left(u_{1}, u_{2}\right) \in N_{\rho}(\Sigma)$ and let $V$ be any vector field on $T^{*}(\Omega)$ equal to $X$ at $\rho$. We define the homogeneous polynomial

AMS (MOS) subject classifications (1970). Primary 35H05, $35 \mathrm{~S} 05$.

Key words and phrases. Pseudo differential operators, classes $L^{m, M}(\Omega, \Sigma)$, symplectic submanifold, winding number. 


$$
c \prod_{h=1}^{M}\left(u_{2}-r_{h} u_{1}\right)=\frac{1}{M !}\left(V^{M} p_{m}\right)_{\rho} .
$$

In view of (2) $\operatorname{Im} r_{h} \neq 0$ for each $h, h=1, \ldots, M$ : let $M^{+}\left(M^{-}\right)$be the number of the $r_{h}$ 's such that $\operatorname{Im} r_{h}>0\left(\operatorname{Im} r_{h}<0\right)$. The integer $N=M^{+}-M^{-}$may take the values $M, M-2, \ldots, 2-M,-M$; here we assume $M \geqslant 2$ and

$$
N=2-M, \text { for every } \rho \text { in } \Sigma .
$$

3. The problem of the hypoellipticity. Let $P \in L^{m, M}(\Omega, \Sigma)$ satisfy (5). We are interested in the following hypoellipticity property:

$$
\begin{aligned}
& \text { For any open subset } U \text { of } \Omega \text { and any distribution } f \text { in } U, P f \in \\
& H_{10 \mathrm{c}}^{s}(U) \text { implies } f \in H_{1 \mathrm{oc}}^{s+m-M / 2}(U) .
\end{aligned}
$$

Let $P$ be the algebraic vector space of all the polynomials in one real variable with complex coefficients and denote by $L(P)$ the space of all the linear maps from $P$ into $P$. We associate to $P$ an application $A_{P}: \rho \in \Sigma \rightarrow A_{P}(\rho) \in L(P)$. The explicit definition of $A_{P}$ will be given in $\S 4$; first let us state our main result.

Theorem 1. Let $P \in L^{m, M}(\Omega, \Sigma)$ satisfy (5). Then (6) holds if and only if

$$
\text { dimension } \operatorname{Ker} A_{P}(\rho)=0 \text {, for every } \rho \text { in } \Sigma \text {. }
$$

4. Definition of $A_{P}(\rho)$. If (5) is satisfied, then it is $M^{+}=1$ and $M^{-}=$ $M-1$ : we will assume $\operatorname{Im} r_{h}<0$ for $2 \leqslant h \leqslant M$ and $\operatorname{Im} r_{1}>0$. As in Helffer [2], initially we construct a family of ordinary differential operators with polynomial coefficients. Consider the symbol $q(x, \xi)$ with asymptotic expansion

$$
\sum_{j=0}^{\infty} q_{m-j / 2} \sim \sum_{t=0}^{\infty} \frac{(-1)^{t}}{t !}\left(\sum \frac{1}{2 i} \frac{\partial^{2}}{\partial x_{s} \partial \xi_{s}}\right)^{t} p .
$$

Using the notations of $\S 2$, we define on $N_{\rho}(\Sigma)$ the polynomial (the leading part coincides with (4))

(8) $c \prod_{n=1}^{M}\left(u_{2}-r_{h} u_{1}\right)+\sum_{\alpha+\beta<M} c_{\alpha, \beta} u_{1}^{\alpha} u_{2}^{\beta}=\sum_{j=0}^{M} \frac{1}{(M-j) !}\left(V^{M-j} q_{m-j / 2}\right)_{\rho}$.

We rewrite the left-hand side of (8) in the symmetric form

$$
\sum_{\gamma(h), h \leqslant M} c_{\gamma(h)}^{\prime} u^{\gamma(n)}
$$

where the components of the multiorder $\gamma(h)=\left(\gamma_{1}, \ldots, \gamma_{h}\right)$ may take the value 1 or $2, c_{\gamma(h)}^{\prime}=c_{\delta(h)}^{\prime}$ if $|\gamma(h)|=|\delta(h)|$ and we have noted

$$
u^{\gamma(h)}=u_{\gamma_{1}} u_{\gamma_{2}} \cdots u_{\gamma_{h}}
$$


Now, maintaining the order of the factors in (10), we replace $u_{2}$ in (9) by $D=$ $-i d / d u_{1}$. We get a differential operator $M(\rho)$ which can be expressed in the form

Set

$$
M(\rho)=c\left(D-r_{M} u_{1}\right) \cdots\left(D-r_{1} u_{1}\right)+\sum_{\alpha+\beta<M} c_{\alpha, \beta}^{\prime \prime} u_{1}^{\alpha} D^{\beta} .
$$

$$
\eta=-i \sum_{\alpha+\beta=M-1} c_{\alpha, \beta}^{\prime \prime} r_{1}^{\beta} / c \prod_{h=2}^{M}\left(r_{1}-r_{h}\right)
$$

We define for $Q \in P$,

$$
\begin{aligned}
A_{P}(\rho) Q\left(u_{1}\right)= & \exp \left(-i r_{1} u_{1}^{2} / 2-\eta u_{1}\right) \\
& \cdot M(\rho)\left[\exp \left(i r_{1} u_{1}^{2} / 2+\eta u_{1}\right) Q\left(u_{1}\right)\right]
\end{aligned}
$$

The definition of $A_{P}(\rho)$ depends on the initial choice of the coordinates $u_{1}, u_{2}$. We can prove that, starting from other canonical coordinates $u_{1}^{\prime}, u_{2}^{\prime}$ and repeating the construction, we get a map $A_{P}^{\prime}(\rho)$ such that $U^{-1}(\rho) A_{P}^{\prime}(\rho) U(\rho)=A_{P}(\rho)$ for some automorphism $U(\rho)$ in $L(P)$. Therefore condition (7) has an invariant meaning.

5. Applications. Take $Q\left(u_{1}\right)=\Sigma_{\nu=0}^{k} b_{\nu} u_{1}^{\nu}$. Developing (13) we obtain

$$
A_{P}(\rho) Q\left(u_{1}\right)=\sum_{\mu=0}^{k+M-2}\left(\sum_{\nu=0}^{k} d_{\mu, \nu} b_{\nu}\right) u_{1}^{\mu}
$$

where $d_{\mu, \nu}$ are polynomials in the variables $c, r_{1}, \ldots, r_{M}, c_{\alpha, \beta}^{\prime \prime}$. We write $D^{(k)}$ for the matrix $\left(d_{\mu, \nu}\right), \mu=0, \ldots, k+M-2, \nu=0, \ldots, k$. Let $\sigma=\left\{\mu_{0}\right.$, $\left.\mu_{1}, \ldots, \mu_{k}\right\}$ be a subset of $\{0,1, \ldots, k+M-2\}$ and let $D_{\sigma}^{(k)}$ denote the minor $\left(d_{\mu_{t}, \nu}\right), t=0, \ldots, k, \nu=0, \ldots, k$. Theorem 1 can be rewritten in the following way.

THEOREM 2. Let $P \in L^{m, M}(\Omega, \Sigma)$ satisfy (5). Then (6) holds if and only if for each fixed $\rho \in \Sigma$ and for every integer $k \geqslant 0$ there exists a subset $\sigma=\left\{\mu_{0}, \mu_{1}, \ldots, \mu_{k}\right\}$ of $\{0,1, \ldots, k+M-2\}$, such that

$$
\operatorname{det} D_{\sigma}^{(k)} \neq 0
$$

A direct computation shows that for $\sigma_{0}=\{M-2, M-1, \ldots, k+M-2\}$

$$
\operatorname{det} D_{\sigma_{0}}^{(k)}=\lambda(\rho) \prod_{\nu=0}^{k}[\ell(\rho)-\nu]
$$

where $\lambda(\rho), \ell(\rho)$ are rational functions of $c, r_{1}, \ldots, r_{M}, c_{\alpha, \beta}^{\prime \prime}: \lambda(\rho) \neq 0$ and $\ell(\rho)$ coincides with the invariant in Boutet de Monvel and Treves [1] and Helffer [3]. 


\section{REFERENCES}

1. L. Boutet de Monvel and F. Trèves, On a class of pseudodifferential operators with double characteristics, Invent. Math. 24 (1974), 1-34. MR 50 \#5550.

2. B. Helffer, Invariants associés à une classe d'opérateurs pseudodifférentiels et applications à l'hypoellipticité, Ann. Inst. Fourier (Grenoble) (to appear).

3. - Sur une classe d'opérateurs hypoelliptiques à caractéristiques multiples, J. Math. Pures Appl. (to appear).

4. J. Sjöstrand, Parametrices for pseudodifferential operators with multiple characteristics, Ark. Mat. 12 (1974), 85-130, MR 50 \#5236.

CENTRE DE MATHÉMATIQUES, ÉCOLE POLYTECHNIQUE, 91120 PALAISEAU, FRANCE

DEPARTMENT OF MATHEMATICS, PRINCETON UNIVERSITY, PRINCETON, NEW JERSEY 08540

Current Address (Luigi Rodino): Istituto Matematico del Politecnico, Corso Duca Degli Abruzzi 24, I-10129 Torino, Italy 